INPLASY

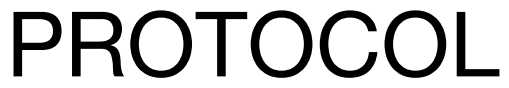

To cite: Zhang et al. Targeted agents in combination with chemotherapy for recurrent ovarian cancer: an updated systematic review and metaanalysis. Inplasy protocol

202140049. doi:

10.37766/inplasy2021.4.0049

Received: 08 April 2021

Published: 08 April 2021

Corresponding author:

Zhang Yinyin

763180639@qq.com

Author Affiliation:

Yi Chun University

Support: Yi Chun University.

Review Stage at time of this submission: Preliminary

searches.

Conflicts of interest:

None declared.

\section{Targeted agents in combination with chemotherapy for recurrent ovarian cancer: an updated systematic review and meta-analysis}

Zhang, $\mathrm{Y}^{1}$; Fan, $\mathrm{HZ}^{2}$.

Review question / Objective: Objectives of this study: On the one hand, the efficacy and safety of combination chemotherapy with targeted drugs (mainly AIS, PARPIS and ICIS) in recurrent ovarian cancer were reassessed by including the latest and most comprehensive data. On the other hand, evidence-based medicine is used to guide clinical practice to provide the best benefit plan for different clinical groups. According to the PICOS principle, "P" refers to recurrent ovarian cancer, "I" refers to chemotherapy combined with targeted drug therapy, "C" refers to chemotherapy alone, "O" refers to objective response rate, progression-free survival, overall survival, incidence of adverse events, and " $S$ " refers to randomized controlled trials. Condition being studied: In order to ensure that the screening process is objective and reliable and to avoid subjective bias, literature screening, selection and quality assessment are independently completed and cross-checked by two researchers according to the pre-established inclusion and exclusion criteria. If there is any discrepancy in the screening process, it will be resolved through negotiation. If there is any discrepancy, it will be decided by the thesis supervisor (a third party).

INPLASY registration number: This protocol was registered with the International Platform of Registered Systematic Review and Meta-Analysis Protocols (INPLASY) on 08 April 2021 and was last updated on 08 April 2021 (registration number INPLASY202140049).

\section{INTRODUCTION}

Review question / Objective: Objectives of this study: On the one hand, the efficacy and safety of combination chemotherapy with targeted drugs (mainly AIS, PARPIS and ICIS) in recurrent ovarian cancer were reassessed by including the latest and most comprehensive data.On the other hand, evidence-based medicine is used to guide clinical practice to provide the best benefit plan for different clinical 
groups.According to the PICOS principle, "P" refers to recurrent ovarian cancer, "I" refers to chemotherapy combined with targeted drug therapy, "C" refers to chemotherapy alone, "O" refers to objective response rate, progression-free survival, overall survival, incidence of adverse events, and " $S$ " refers to randomized controlled trials.

Condition being studied: In order to ensure that the screening process is objective and reliable and to avoid subjective bias, literature screening, selection and quality assessment are independently completed and cross-checked by two researchers according to the pre-established inclusion and exclusion criteria. If there is any discrepancy in the screening process, it will be resolved through negotiation. If there is any discrepancy, it will be decided by the thesis supervisor (a third party).

\section{METHODS}

Participant or population: Patients with ovarian cancer diagnosed by histopathology (recurrence criteria refer to the efficacy evaluation criteria for solid tumors -- RECIST) who relapse after achieving clinical complete remission with ideal tumor cell reduction and standard platinum-based chemotherapy.

Intervention: Chemotherapy combined with targeted drug therapy.

\section{Comparator: Pure chemotherapy.}

Study designs to be included: Randomized Controlled Trial (RCT).

Eligibility criteria: Patients with ovarian cancer diagnosed by histopathology (recurrence criteria refer to the efficacy evaluation criteria for solid tumors -RECIST) who relapse after achieving clinical complete remission with ideal tumor cell reduction and standard platinum-based chemotherapy.

Information sources: Computer retrieval comprehensive Chinese knowledge resource pool (CNKI), the data of ten thousand academic papers in the pool, VIP, Science and technology periodical database (VIP), the Chinese biomedical literature database (CBM), PubMed, Embase, the Cochrane library, Web of Science, clinical trial registry (China + the United States), and other nine academic database and baidu, bing and Google academic search platform,And manually searched the "Progress of Modern Obstetrics and Gynecology", "International Journal of Obstetrics and Gynecology", "Chinese Journal of Modern Obstetrics and Gynecology", "Practical Obstetrics and Gynecology" and other obstetrics and gynecology journals.

Main outcome(s): Objective response rate (ORR), progression-free survival (PFS), overall survival (OS), incidence of adverse reactions.

Quality assessment / Risk of bias analysis: The quality of the included studies was assessed using the Cochrane Risk Bias Assessment Tool and the modified Jadad Scale.

Strategy of data synthesis: The data included in this study were processed using RevMan5.3 and Stata 13.1.Survival data, i.e., time-event data (overall survival and progression-free survival), were combined with hazard ratios (HR) for each study using inverse variance method.Enumeration data, i.e., binary data (objective remission rate, incidence of adverse events), were extracted from the number of patients in the trial group and the control group and the total number of patients, respectively. M-H method was selected and the relative risk (RR) was used as effect size to combine the studies. When $P>0.1$ and $12<50 \%$, there was no heterogeneity, and the effect size was combined with fixed effect. When $P<0.1$ and $12 \geq 50 \%$, heterogeneity is suggested, and the cause of heterogeneity should be found through subgroup analysis or sensitivity analysis or Meta regression. Fixed effects model (FEM) was used for heterozygous exclusion, and random effects model (REM) was used for 
heterozygous exclusion if heterogeneity could not be excluded.

Subgroup analysis: Subgroup analyses were performed for disease type (platinumsensitive or platinum-resistant), chemotherapy choice (first-line or secondline), and ethnic difference (East Asian or Caucasian).

Sensitivity analysis: Any literature can be deleted by RevMan software to observe whether there is any change in the total effect size. If there is no significant difference, the calculation results are stable and reliable. Sensitivity maps can be drawn using the Stata software to determine the sensitivity based on the estimated value of each study site in the $95 \% \mathrm{Cl}$ distribution of the total effect size. Subgroup analysis (examining intergroup heterogeneity or intra-group heterogeneity) can be performed for significantly heterogeneous results.

Country(ies) involved: China.

Keywords: Targeted therapy; Recurrent ovarian cancer; Effectiveness; Security; Meta analysis.

Contributions of each author:

Author 1 - ZHANG YINYIN.

Author 2 - FAN HZ. 